\title{
Chondrogenic Differentiation of Adipose-Derived Stem Cells by Radiofrequency Electric Stimulation
}

María Luisa Hernández-Bule ${ }^{1^{*}}$, María Ángeles Trillo ${ }^{1}$, María Ángeles Martínez-García ${ }^{2}$, Carlos Abilahoud ${ }^{3}$ and Alejandro Úbeda ${ }^{*}$

${ }^{1} B E M-R e s e a r c h$ Service, Ramón y Cajal University Hospital - IRYCIS, Madrid, Spain

${ }^{2}$ Department of Endocrinology and Nutrition, Ramón y Cajal University Hospital - IRYCIS and CIBERDEM Biomedical Research Center, Madrid, Spain

${ }^{3}$ Department of Electrical Engineering, E.T.S. of Engineering and Industrial Design, Polytechnic University of Madrid, Madrid, Spain

\section{Abstract}

Objective: Although capacitive-resistive electric transfer (CRET) therapies, based on transdermal application of electrothermal radiofrequency currents, have shown promising therapeutic effectiveness in regeneration of traumatic or degenerative tissue lesions, their potential effects on tissues like cartilage, having poor regenerative capabilities, have not been studied sufficiently. Here we investigate the effects of the exposure to a $448 \mathrm{kHz}$ current typically used in CRET therapy, on the early chondrogenic differentiation of human, adipose-derived stem cells (ADSC).

Materials and methods: Stem cells obtained from healthy donors were differentiated in chondrogenic medium for 16 days. During the last 2 days of incubation the cultures were intermittently exposed or sham-exposed to a 448$\mathrm{kHz}$, sine wave current, administered at a $50 \mu \mathrm{A} / \mathrm{mm}^{2}$ subthermal density. The cellular response was assessed by: XTT proliferation assay, glycosaminoglycans (GAG) and collagen quantification (image analysis, Blyscan assay and immunoblot) and analysis of the expression of chondrogenic factors Sox5 and Sox6, and of the transcription factor ERK1/2 and its active form p-ERK1/2 (immunoflorescence, immunoblot and RT-PCR).

Results: The electric stimulus significantly increased the levels of both, cartilage-specific collagen type II and GAG in the extracellular matrix of the differentiating cultures. Although no changes were observed in the expression of the SOX genes at the end of the 48-hour treatment, the stimulus did induce significant overexpression of transcription factors L-Sox5, Sox6 and p-ERK1/2. Since these proteins are crucial regulators of the synthesis of the extracellular matrix during chondrogenic differentiation, it is likely that their overexpression is involved in the observed increases in the content of extracellular collagen and GAG.

Conclusion: The present data set provides support to the hypothesis that the electric component of the electrothermal treatment applied in CRET therapies could stimulate cartilage repair by promoting chondrogenic differentiation. These data, coupled with previously reported results that in vitro treatment with the same type of subthermal electric signal promotes proliferation of undifferentiated ADSC, identify molecular phenomena underlying the potential repairing and regenerative effects of such radiofrequency currents.

Keywords: Electrotherapy; Radiofrequency; Chondrogenesis; Extracellular matrix; Sox5, Sox6; Collagen type II

\section{Introduction}

There is ample evidence that stimulation with electric currents and electric and/or magnetic fields can induce a variety of cellular and molecular responses, including microfilament reorganization [1], redistribution of cell surface receptors [2] or cell migration [3], as well as changes in intracellular calcium dynamics [4] and in stem cell proliferation or differentiation [5-7]. This body of evidence has provided indications that some electric and/or magnetic stimuli may exert favourable effects in the control of cell and tissue homeostasis, thus intervening in tissue repair and regeneration processes. Indeed, electrotherapy has been successfully applied to bone fracture consolidation [8], soft tissue regeneration [9], nerve fibre repair [10] or treatment of cancerous lesions [11]. Electric and electromagnetic therapies have proven also effective in the treatment of osteoarticular lesions such as osteoarthritis $[12,13]$ or degenerative disc disease $[14,15]$. Similarly, capacitive-resistive electric transfer (CRET) electrothermal therapies, based on non-invasive application of radiofrequency (RF) electric currents, have been used successfully in regeneration of muscle [16-19], tendons [16,19-21] and ligaments [22].

As for cartilage and other tissues having poor capacity for regeneration and cellular self-renewal [23], although the potential repairing effects of electrotherapies remain a matter of debate, it has been reported that exposure to electric or electromagnetic stimulation can induce in articular chondrocytes cellular responses involved in prevention of degenerative damage [24]. Evidence of this kind has served as a basis for proposing that stimulation with specific electric and/or magnetic parameters could favour cartilage regeneration through promotion of extracellular matrix protein synthesis and/or of chondrocyte or prechondrocyte proliferation $[25,26]$. The mechanisms underlying these effects would involve electrical stimulation of cell membrane receptors which, through activation of signalling molecules, would trigger a cascade of effects resulting in cellular migration, proliferation or differentiation $[27,28]$. In fact, evidence exists that stem cells present in the cartilaginous tissue could be a plausible target for treatment with electric fields or currents. Indeed, stimulation with $500 \mathrm{mV} / \mathrm{mm}$, direct current electric field has been reported to promote survival of grafted neural stem cells, guiding

*Corresponding author: M Luisa Hernández-Bule, Servicio BEM-Investigación Hospital Universitario Ramón y Cajal - IRYCIS, 28034 Madrid, Spain, Tel: +34913581365; +34-913368699; E-mail: mluisa.hernandez@hrc.es

Received December 19, 2017; Accepted December 22, 2017; Published December 29, 2017

Citation: Hernández-Bule ML, María Trillo Á, Martínez-García MÁ, Abilahoud C, Úbeda A (2017) Chondrogenic Differentiation of Adipose-Derived Stem Cells by Radiofrequency Electric Stimulation. J Stem Cell Res Ther 7: 407. doi: 10.4172/2157-7633.1000407

Copyright: (c) 2017 Hernández-Bule ML, et al. This is an open-access article distributed under the terms of the Creative Commons Attribution License, which permits unrestricted use, distribution, and reproduction in any medium, provided the original author and source are credited. 
their migration and stimulating their differentiation and functioning within the lesion [29]. Also, time-varying electric fields $(60 \mathrm{kHz}, 20 \mathrm{mV} /$ $\mathrm{cm})$ and pulsed electromagnetic fields $(27.1 \mathrm{MHz})$ have been reported to promote osteogenic differentiation of mesenchymal stem cells [30,31]. Previous results by our group have shown that intermittent exposure to subthermal densities of RF $(448 \mathrm{kHz})$ currents of the type applied in CRET therapies promotes proliferation in undifferentiated cultures of adipose-derived stem cells (ADSC) obtained from healthy donors [6]. Such proliferative response did not affect the subsequent ability of the ADSC to normally differentiate towards chondrocyte, adipocyte or osteocyte lineages when the cultures were supplemented with the corresponding differentiating factors. Our studies also revealed that in vitro exposure to the above subthermal RF currents can modulate the expression of genes controlling the synthesis and expression of proteins intervening in early stages of the adipogenic differentiation of ADSC [7].

Based on the above experimental evidence, the possibility can be posed that CRET currents could also be effective in modulating processes intervening in cartilage regeneration, through stimulation of stem cells present in the damaged or degenerating tissue. Thus, the aim of the present study was to investigate the potential action of the in vitro electrostimulation with subthermal pulses of CRET current on early chondrogenic differentiation of ADSC. The cellular response was assessed by analysis of cell proliferation, quantification of extracellular matrix components synthesized during chondrogenesis, analysis of gene and protein expression of the chondrogenic markers L-Sox5 and Sox6, and assessment of the activation of the Mitogen-Activated Protein Kinase Extracellular Signal-Regulated Kinases 1 and 2 (MAPK ERK1/2) signaling pathway, which has proven an important regulator of cartilage-specific gene expression in a variety of chondroprogenitors and chondrogenic cell types [32].

\section{Material and Methods}

\section{Cell culture}

ADSC were isolated from subcutaneous adipose tissue surgically obtained from 4 healthy donors: two men, 65 and 69 years old, and two women of 28 and 35. This protocol, which has been described in detail in previous studies [6], met the ethical standards applicable in the European Union, and was approved by the ethics committee for clinical trials of Hospital Universitario Ramón y Cajal. Briefly, ADSC were isolated from $0.5-1 \mathrm{~cm}^{3}$ pieces of fat and sliced into $1-2 \mathrm{~mm}^{3}$ fragments which were subsequently digested with $1 \mathrm{mg} / \mathrm{ml}$ collagenase A (Roche Applied Science, Basel, Switzerland) and centrifuged to isolate the vascular-stromal fraction. The resulting pellet was resuspended in culture medium (MesenPro-RSTM, Gibco, Invitrogen, Camarillo, CA, USA) supplemented with $1 \%$ glutamine (Gibco) and $1 \%$ penicillinstreptomycin (Gibco), and the cells were seeded in a $75 \mathrm{~cm}^{2}$ T-flask (Falcon, Corning incorporated, Life Sciences, Durham, NC, USA). After 4 days the culture medium was renewed, and 3 days after, when confluent, the cells were subcultured. Flow cytometry analysis of expression of characteristic markers of multipotential mesenchimal cells, CD29, CD44, CD73, CD90 and CD105 was conducted. The results confirmed that the ADSC were positive for all these markers (see supplementary information).

\section{Chondrogenic differentiation}

Preliminary tests revealed that in our model of electrical stimulation, the RF current distribution within the Petri dish and in the plated cells is influenced by the culture type. Namely, cells forming multi-cellular, spheroidal structures or micromasses were found to be less sensitive to the electrical treatment than those adopting a monolayer distribution on the dish surface (data not shown). This would be attributable to the fact that monolayer configuration allows homogenous exposure of all cells in the culture to the electrical stimulus, whereas when grouped into three-dimensional micromasses with relatively high electrical resistivity, the stimulus would reach only those cells located at the outermost layer of the spheroid. This methodological requirement, together with the fact that monolayer culture has been reported advantageous to chondrocyte differentiation within the first three weeks of incubation $[33,34]$ led us to adopt monolayer culture as a suitable model for studying the early chondrogenic response to RF electrostimulation.

ADSC in passages 3 to 6 were seeded in $60 \mathrm{~mm}$ Petri dishes (Nunc, Roskilde, Denmark) at a density of 2270 cells $/ \mathrm{cm}^{2}$. The cells were plated directly on the bottom of the dish, except for immunofluorescence assays and Alcian blue staining, in which the cells were seeded on glass coverslips placed on the bottom of the plate. A total of 12 Petri dishes were used in each experimental run. At day four after platting, 8 of the dishes were incubated in chondrogenic differentiating medium, composed of high-glucose D-MEM (Biowhittaker, Lonza, Verviers, Belgium) supplemented with $10 \%$ inactivated foetal bovine serum (Gibco), 1\% glutamine and 1\% penicillin-streptomycin (Gibco), 37.5 $\mu \mathrm{g} / \mathrm{ml}$ ascorbic acid-2 phosphate (Sigma), $10 \mathrm{ng} / \mathrm{ml} \mathrm{TGF-} \beta 1$ (Peprotech, Rocky Hill, NJ, USA), $10 \mu \mathrm{g} / \mathrm{ml}$ insulin and $39.25 \mu \mathrm{g} / \mathrm{ml}$ dexamethasone (Sigma). During the last $48 \mathrm{~h}$ of incubation in this chondrogenic medium, the cultures were RF- or sham-exposed. The remaining 4 dishes were incubated in basal medium composed of high-glucose D-MEM (Biowhittaker, Lonza, Verviers, Belgium) supplemented with $10 \%$ inactivated foetal bovine serum (Gibco), $1 \%$ glutamine and $1 \%$ penicillin-streptomycin (Gibco).

\section{Electric treatment}

The radiofrequency exposure procedure has been described in detail elsewhere [6]. Briefly, the exposure was carried out by means of pairs of sterile stainless steel electrodes designed ad hoc for in vitro stimulation. All electrode pairs were connected in series to a signal generator (model Indiba Activ HCR 902, INDIBA, Barcelona, Spain), though only those inserted in the RF-exposed samples were energized. The stimulation pattern consisted of 5-minute pulses of $448 \mathrm{kHz}$, sine wave current at a subthermal density of $50 \mu \mathrm{A} / \mathrm{mm}^{2}$, separated by $4-\mathrm{h}$ interpulse lapses, for a total period of $48 \mathrm{~h}$. Such exposure parameters have shown to affect human ADSC proliferation and differentiation in previous studies by our group [6,7]. In the present study, after 14 days of incubation in chondrogenic differentiating medium (CD, 8 dishes per experimental run) or in non-differentiating basal medium (ND, 4 dishes per run), electrode pairs were fitted inside all 12 Petri dishes. The electrodes were energized during 48 hours with CRET current in 4 of the samples incubated in differentiating medium (CD+CRET), but not in the remaining 8 dishes, $\mathrm{CD}$ and $\mathrm{ND}$ sham-exposed controls.

\section{XTT proliferation assay}

Cell proliferation of cultures in passages 3 to 7 was determined by XTT assay (Roche). After $48 \mathrm{~h}$ of CRET- or sham- treatment, the cells were incubated for 3 hours with the tetrazolium salt XTT in a $37^{\circ} \mathrm{C}$ and $6.5 \% \mathrm{CO}_{2}$ atmosphere. The metabolically active cells reduced XTT into coloured formazan compounds that were quantified with a microplate reader (TECAN, Männedorf, Switzerland) at a $492 \mathrm{~nm}$ wavelength. The obtained colorimetric values correlated directly with the number of active cells. A total of 3 experimental replicates were conducted. 
Citation: Hernández-Bule ML, Trillo MÁ, Martínez-García MÁ, Abilahoud C, Úbeda A (2017) Chondrogenic Differentiation of Adipose-Derived Stem Cells by Radiofrequency Electric Stimulation. J Stem Cell Res Ther 7: 407. doi: 10.4172/2157-7633.1000407

Page 3 of 10

\section{Image segmentation for quantitative assessment of collagen content}

The total collagen content in the cultures was evaluated by Light green staining. Cells grown on coverslips were fixed with $4 \%$ paraformaldehyde, and their nuclei and collagen matrix were stained with Harris' hematoxylin (Merk) and $0.2 \%$ Light green (SigmaAldrich), respectively. Bright-field micrographs were taken with a $1280 \times 1024 \times 24$ Nikon DS-Ri2 digital camera attached to a Nikon Eclipse TE300 microscope. AnalySIS 3.1 software (Soft Imaging Systems GmbH, Münster, Germany) was used for data acquisition. Three replicates were conducted of each experiment and a total of 150 images were processed: 60 images of the non-differentiated group, 43 of the control group incubated in chondrogenic medium and shamstimulated, and 47 of the group incubated in chondrogenic medium and RF-stimulated. The chromatic information in the images allows segmenting collagen with respect to other photographed structures. Figure 1a shows a representative micrograph acquired according the described procedure, portraying ADSC in an early stage of chondrogenic differentiation and the extracellular matrix synthesized by these cells. Collagen is green stained, while cytoplasms and cell nuclei are purple stained, being the nuclei paler. The original images were processed through a collagen segmentation method which uses the Matlab R2013a platform. This method reduces the image resolution by $60 \%$ in order to optimize the segmentation time. Next, the image noise was filtered to maintain the average intensity of the images within a 130-160 pixel range. The RGB colour obtained image was converted to the LAB system (Figure 1b) by using the RGB2LAB algorithm [35], which applies the "decorrstretch" function to exclude pixels within the red-purple colour spectrum and generates a negative greyscale image. This image was binarized assigning a zero (0) value to those pixels with intensities below 0.29 and discarding them (Figure 1c). Then, the remaining pixels, with value 1 , were converted back to their original colour and the "decorrstretch" function was reapplied, allowing accurate collagen segmentation (Figure 1d). Once collagen was segmented (Figure 1e), the method determined the number of pixels and converted the green colour tonalities in greyscale according to their intensity, which is directly proportional to the collagen amount. Finally, to optimize the analysis, a negative image was obtained showing the collagen in greyscale on a black background (Figure 1f). From this segmentation, the amount and intensity of pixels in each image was computer quantified, and a comparative statistical analysis of the data corresponding to the different experimental groups was carried out.

\section{Alcian blue quantification for assessment of glycosaminoglycan content}

RF- and sham-exposed samples grown on coverslips were fixed in paraformaldehyde and stained with a stock solution of $1 \%$ Alcian blue (BDH, Poole, UK) in 3\% acetic acid. Photomicrographs were taken of the cultures and the images were computer analyzed as described above. The glycosaminoglycan (GAG) content was determined by quantification of Alcian blue positive staining using AnalySIS 3.1 software. Four replicates were carried out per experiment, and a total of 280 micrographs of cell cultures grown in chondrogenic medium were processed: 140 images of sham-stimulated controls and 140 of RFexposed samples.

\section{Blyscan assay for glycosaminoglycan quantification}

The RF- or sham-exposed cells were resuspended in a digestion solution containing $0.2 \mathrm{M}$ sodium phosphate buffer $(\mathrm{pH}=6.4), 0.1$ $\mathrm{M}$ sodium acetate, $0.01 \mathrm{M}$ EDTA, $5 \mathrm{mM}$ cysteine- $\mathrm{HCl}$ (Sigma) and $0.2 \mathrm{mg} / \mathrm{ml}$ Papain (Roche), and were incubated overnight at $65^{\circ} \mathrm{C}$. The GAG content was quantified by Blyscan assay following the manufacturer's instructions (Biocolor, UK). Absorbance was read in a spectrophotometer (Cecil CE 2021; UK) at a $656 \mathrm{~nm}$ wavelenght. GAG concentration in the samples was assessed through the GAG standard curve and total GAG was normalized over DNA content. The DNA
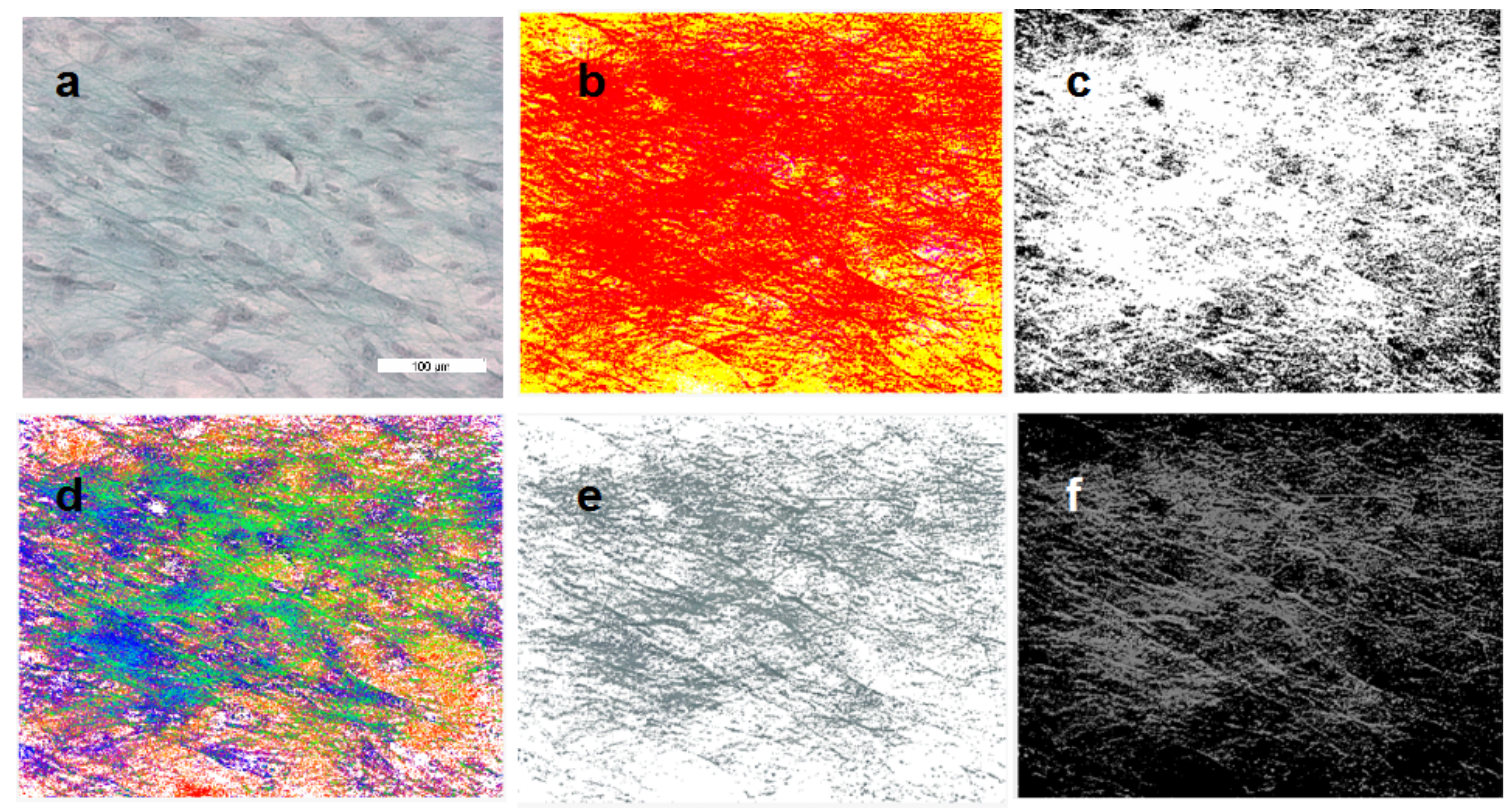

Figure 1: Computational method for quantification of collagen content in micrographic images. (a) Original image of a monolayer culture showing green-stained collagen fibers. (b)The RGB color image is converted to LAB color system. (c) Binarization. (d) Decorrstretch. (e) Segmented collagen. (f) Negative of the previous image, displaying collagen in greyscale. 
concentration was determined with bisBenzimide (33258 dye Sigma), using salmon sperm DNA solution to generate the standard curve. Excitation (at $360 \mathrm{~nm}$ ) and emission (at $465 \mathrm{~nm}$ ) levels were quantified using a microplate reader (TECAN, Männedorf, Switzerland).

\section{Immunofluorescence for L-Sox 5 and Sox6}

Transcription factors Sox 5 and Sox6 act as regulators of cartilage development by boosting the activation of genes such as Coll1a2, Col2al and Agcl which control the synthesis of chondrocyte-specific extracellular matrix proteins [36]. To evaluate the effects of the electric stimulation on the expression of L-Sox 5 (the long isoform of protein Sox5) and Sox6, the cultures were seeded on coverslips and RF- or shamexposed as described above. Subsequently, the samples were fixed with $4 \%$ paraformaldehyde and incubated overnight at $4^{\circ} \mathrm{C}$ with anti-L-Sox 5 polyclonal antibody (1:50, Santa Cruz Biotechnologies, Texas, USA) and anti-Sox6 polyclonal antibody (1:50, Santa Cruz Biotechnologies). Afterwards, the samples were fluorescence stained with Alexa Fluor ${ }^{\circ}$ 488 goat anti-rabbit IgG (Molecular Probes, Eugene, Oregon, USA) for $1 \mathrm{~h}$ at room temperature. The cell nuclei were counterstained with bisBenzimide $\mathrm{H}$ 33258. Photomicrographs were taken and analyzed as described above. Three replicates of each experiment were performed and a total of 540 images were processed: 180 images of the samples incubated in the absence of differentiating medium (ND) and 180 of each of the two groups grown in chondrogenic medium: electrically stimulated samples (CD+CRET) and sham-exposed controls (CD).

\section{Immunoblot for L-Sox5, Sox6, ERK, p-ERK1/2 and collagen type II}

The immunoblot procedure has been described in detail elsewhere [37]. Briefly, the blots were incubated overnight in anti-L-Sox 5 polyclonal antibody (1:1200, Santa Cruz Biotechnologies), anti-Sox6 polyclonal antibody (1:100, Santa Cruz Biotechnologies), anti-pERK1/2 polyclonal antibody (1:500, Cell Signalling, Danvers, MA, USA), anti-ERK1/2 polyclonal antibody (1:1000, Invitrogen, USA) and anti-collagen II polyclonal antibody (1:1000, Sigma-Aldrich, China). Anti- $\beta$-actin monoclonal antibody (1:5000, Sigma-Aldrich, Israel) was used as loading control. The membranes were incubated for one hour at room temperature with anti-rabbit IgG conjugated to IRdye $800 \mathrm{CW}$ (1:10000, LI-COR Biosciences, Nebraska, USA) and with anti-mouse IgG conjugated to IRdye 680 LT (1:15000, LI-COR Biosciences). Then, the membranes were scanned with a LI-COR Odyssey scanner (LICOR Biosciences). When ECL-chemiluminescence was required, the membranes were incubated with ECL-anti-mouse IgG horseradish peroxidase-linked antibody (GE Healthcare, Little Chalfont, Buckinghamshire, UK) or with ECL-anti-rabbit IgG horseradish peroxidase-linked antibody (GE Healthcare). The obtained bands were densitometry evaluated (PDI Quantity One 4.5.2 software, BioRad). At least five experimental replicates were conducted for each protein. All values were normalized over the loading control.

\section{RT-PCR for the SOX5 and SOX6}

RT-PCR assay was applied for studying the potential influence of the RF stimulus on SOX5 and SOX6 gene expression. Total RNA was isolated using an RNeasy Lipid Tissue Mini Kit (Qiagen, Germantown, MD, USA) according to the manufacturer's instructions. The concentration and quality of the extracted RNA were assessed by measuring the $260 / 280$ and $260 / 230$ absorbance ratios with a NanoDrop 1000 spectrophotometer (Thermo Scientific, Wilmington, DE, USA). First-strand cDNA was synthesized with SuperScript VILOMasterMix (Invitrogen by Life Technologies, USA) using an equal amount of total RNA and following the manufacturer's instructions. Prevalidated TaqMan Gene Expression assays were applied for relative quantification of SOX5 (Assay ID Hs00753050_s1, Applied Biosystems) and SOX6 (Assay ID Hs00264525_m1, Applied Biosystems), using a StepOnePlus Real-Time PCR System (Applied Biosystems). Human GAPD (GAPDH) Endogenous control (Applied Biosystems) was used to normalize the expression levels of the target genes in each sample. A threshold cycle $(\mathrm{Ct})$ was obtained for each amplification curve, and a $\Delta \mathrm{Ct}$ value was first calculated by subtracting the $\mathrm{Ct}$ value for GAPDH cDNA from the $\mathrm{Ct}$ value of the specific transcript. Data were expressed in arbitrary units and normalized over the differentiated, sham-exposed controls, using the following transformation: $\Delta \mathrm{Ctx}-\Delta \mathrm{CtCD}=2-\Delta \Delta \mathrm{Ct}$. RNA from mature chondrocytes (DV Biologics, CA, USA) was used as positive control of expression. A total of six experimental replicates, with four dishes per experimental group, were processed. Each sample from four pooled dishes per experimental condition was processed in triplicate. Negative controls with no RT enzyme were included in all reactions.

\section{Statistical analysis}

All procedures and analyses were conducted in blind conditions for RF- or sham-exposure and for presence or absence of chondrogenic medium. Three or more independent replicates were conducted per experiment, the results being expressed as means \pm standard deviation (SD) or means \pm standard error of the mean (SEM). The data obtained by digital segmentation of images were analyzed using the StatGragphics XVII.1 software, providing the average values of IPixeles (number of pixels multiplied by their average intensity) corresponding to the segmented collagen. An ANOVA one-way test was applied, followed by Fisher's Least Significant Difference (LSD) test. The statistical method was validated by confirming the homoscedasticity of the residuals, which follow a normal distribution. For the rest of the experiments the one-way ANOVA test was applied, followed by two-tailed unpaired Student's t-test and/or Bonferroni post-test, using Graph-Pad Prism software (GraphPad Software, San Diego, CA, USA). Differences $\mathrm{p}<0.05$ were considered significant statistically.

\section{Results}

\section{RF effects on cell proliferation}

The results of the XTT assay summarized in Figure 2 show that compared to samples incubated for 16 days in basal medium (ND), incubation in chondrogenic medium (CD) induced a statistically significant decrease in cell proliferation. Such antiproliferative response was slightly, but significantly increased by exposure to RF during the last 48 hours of incubation in differentiating medium (CD vs. CD+CRET).

\section{RF effects on collagen content}

The image segmentation analysis of Light green stained cultures revealed that, compared with undifferentiated cultures (ND), incubation in chondrogenic medium induced a $26 \%$, statistically significant increase in the average collagen content (CD; Figure 3a and $3 \mathrm{~b}$ ). As for samples exposed to RF during the last two days of chondrogenic differentiation (CD+CRET), they showed a $23 \%$ average increase, statistically significant, in collagen content over their shamexposed controls, CD. The results of the immunoblot densitometry (Figure $3 \mathrm{c}$ and $3 \mathrm{~d}$ ) revealed that a significant proportion of the collagen synthesized in response to the electrical stimulus corresponded to cartilage-specific type II collagen (51\% over differentiated controls, CD). 


\section{RF effects on glycosaminoglycan content}

The effects of CRET exposure on GAG content was estimated through image analysis quantification of Alcian blue staining and by Blyscan assay. The analysis of the micrographic images revealed

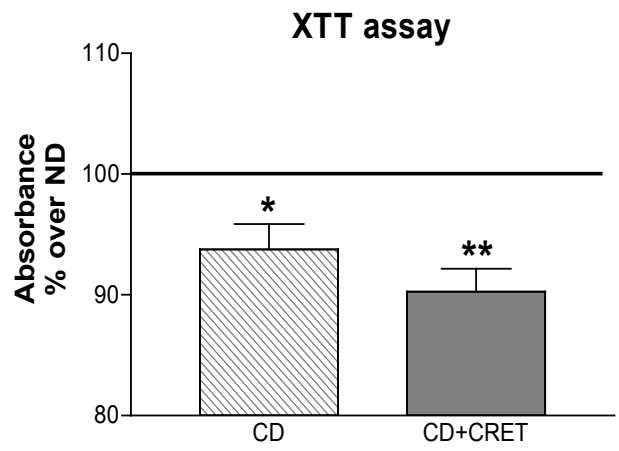

Figure 2: XTT proliferation assay. CD: differentiated cells, grown in chondrogenic differentiating medium for 16 days post-seeding and sham-exposed to CRET during the last $48 \mathrm{~h}$. CD + CRET: cells incubated in chrondrogenic differentiating medium for 16 days and exposed to CRET during the last $48 \mathrm{~h}$. Data are means \pm SEM normalized over those of samples grown in basal medium for 16 days and sham-exposed to CRET during the last $48 \mathrm{~h}$ (ND). Three repeats per experimental group ${ }^{*}: 0.01 \leq p<0.05 ;{ }^{* *}: 0.001 \leq p<0.01$; Student's t test. that incubation in the presence of differentiating medium (CD) increased the amount and condensation of GAG with respect to that in undifferentiated cultures kept in basal medium (ND; Figure 4a). Computerized Image analysis also showed that, compared to shamexposed differentiated controls (CD), stimulation with CRET during the last $48 \mathrm{~h}$ of incubation in chondrogenic medium significantly increased (10\%) the average levels of GAG labeling (Figure $4 \mathrm{~b}$ ). These results were consistent with those from the Blyscan assay, which revealed a $20 \%$, statistically significant increase with respect to the sham-exposed controls, in the average GAG content after electrical stimulation of differentiating samples (Figure $4 b$ ).

\section{RF effects on L-Sox5 and Sox6: immunofluorescence assay}

Presence of nuclear labelling for the proteins L-Sox 5 and Sox6, involved in early chondrogenic differentiation, was observed in all experimental groups (Figure 5a). Indeed, even the control cultures maintained in standard, non-differentiating medium showed traces of this type of labeling, since typically a small proportion of ADSC can spontaneously pre-differentiate into chondrocytes and other cell types [7]. Compared with sham-exposed cultures grown in chondrogenic medium (CD) the RF stimulated samples (CD+CRET) showed significantly increased nuclear labeling for L-Sox 5 and Sox6 (16\% and $44 \%$, respectively; Figure $5 b$ ). By contrast, incubation of sham-exposed controls in chondrogenic medium did not induce significant changes in the labeling of these transcription factors (ND vs. CD).

\section{a}
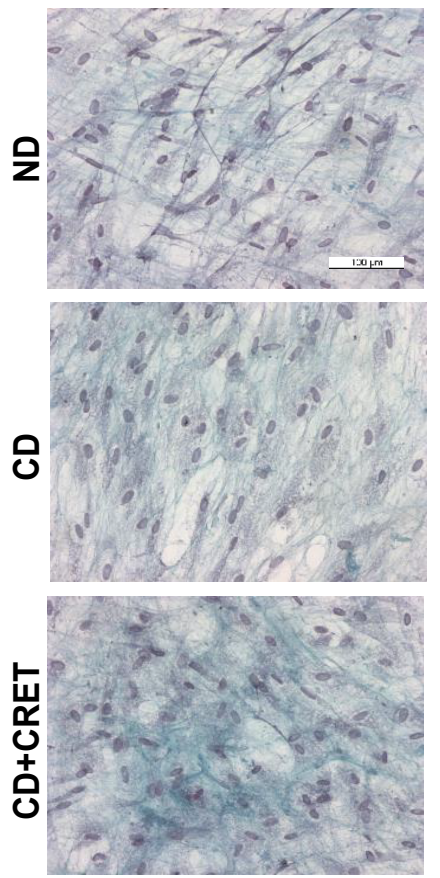

b

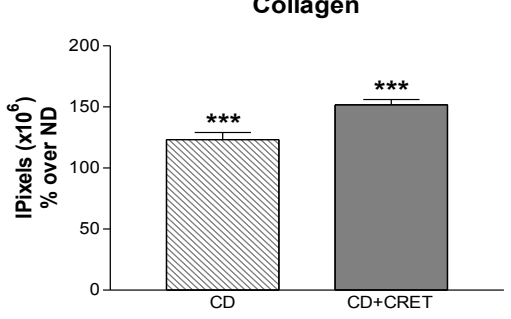

C
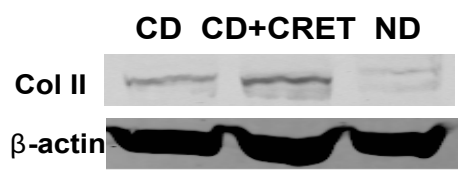

d

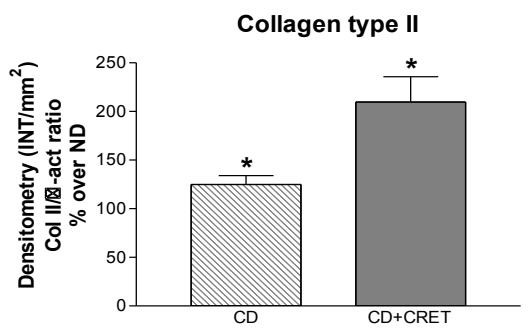

Figure 3: Quantification of collagen content. (a) Collagen staining. Representative micrographs of cultures stained with Light green and Harris' hematoxylin. ND, $\mathrm{CD}$ and CD+CRET: same notations as in Figure 2; Bar: $100 \mu \mathrm{m}$. (b) Computerized quantification of collagen content. IPixels: number of pixels multiplied by their intensity. Data are means \pm SD of $\mathrm{N}=40$ to 60 images per experimental group, out of a total of 150 images obtained from 3 experimental replicates of each group. Data normalized over sham-exposed, non-differentiated controls ND. ${ }^{* *}: p<0.001$; ANOVA and Fisher's LSD tests. (c) Representative blots for collagen type II expression; $100 \mu \mathrm{g}$ protein per lane. (d) Collagen type II expression in CRET-exposed samples, normalized over sham-exposed, non-differentiated controls ND. Data are means \pm SD of 5 repeats per experimental group. Collagen type II/ $\beta$-actin ratio; INT/mm²: Intensity per $\mathrm{mm}^{2}{ }^{*}{ }^{*}: 0.01 \leq p<0.05$; Student's $t$ test. 
a
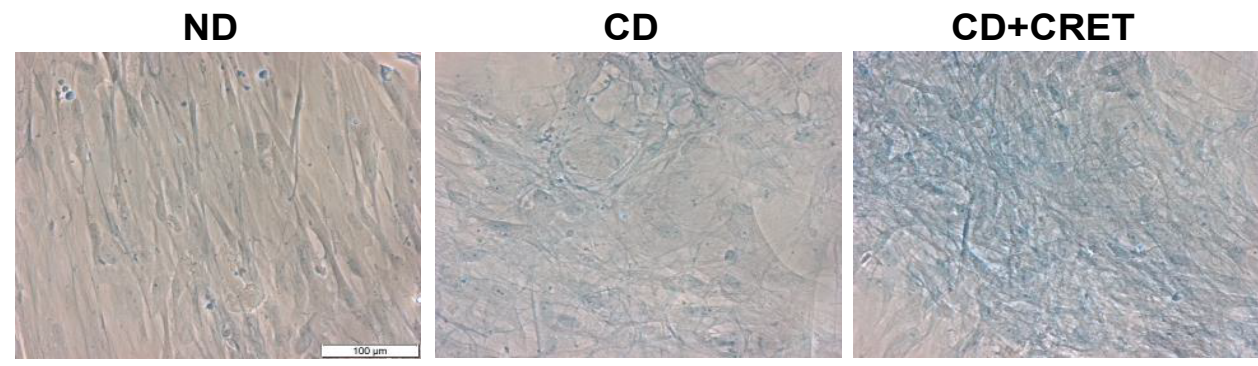

Glycosaminoglycans

b

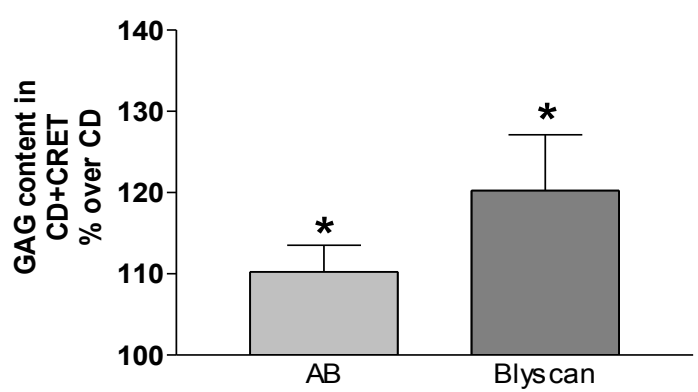

Figure 4: Quantification of GAG content. (a) GAG staining in ADSC cultures grown for 16 days post-plating and Alcian blue stained for GAG labeling. ND, CD and CD+CRET: same notation as in previous figures. Bar: $100 \mu \mathrm{m}$. (b) GAG quantification. AB: image analysis of the Alcian blue intensity incorporated by the cultures. Blyscan: quantification of GAG/DNA ratio by Blyscan assay. Data are mean \pm SEM values in CRET-exposed samples, normalized over those in the corresponding sham-exposed, differentiated controls (CD). Between 4 and 6 experimental replicates per assay. ${ }^{*} 0.01 \leq p<0.05$; Student's $t$ test.

a
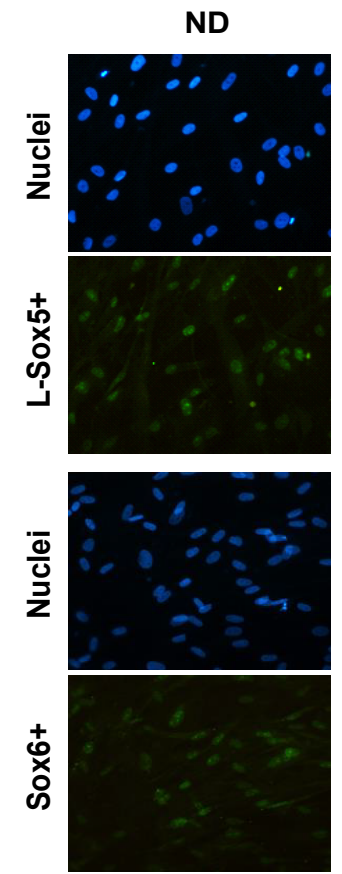

CD
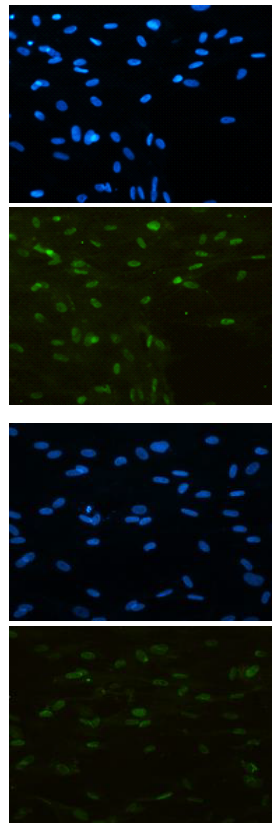

\section{CD+CRET}
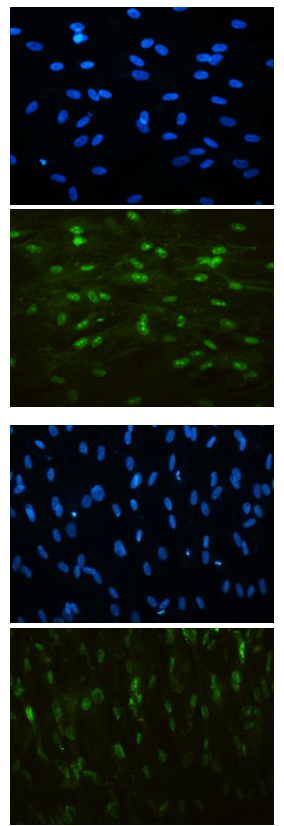

b
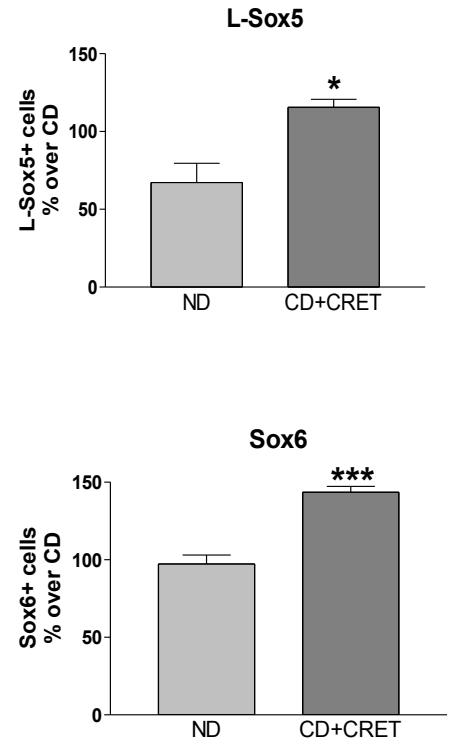

Figure 5: Immunofluorescence for L-Sox5 and Sox6. (a) Representative immunofluorescence images for L-Sox5 and Sox6. ND, CD and CD+CRET: same notation as in previous figures. Bar: $100 \mu \mathrm{m}$. (b) Image analysis quantification of L-Sox5+ and Sox6+ labeled cells. Data are mean \pm SEM values, normalized over those in the corresponding sham-exposed differentiated controls (CD). Between 4 and 6 experimental replicates per assay. ${ }^{*} 0.01 \leq p<0.05 ;{ }^{* \star *} p<0.001 ;$ Student's $t$ test. 


\section{RF effects on L-Sox5, Sox6, ERK1/2 and p-ERK1/2 expression:} immunoblot assay

Electric stimulation significantly increased the expression of factors L-Sox $5(25 \%)$ and Sox6 (20\%) with respect to that in sham-exposed controls ND and CD (Figure 6a and 6b). As for factor ERK1/2, its expression was not significantly affected, either by the chondrogenic medium or by electrostimulation, whereas its active form, factor p-ERK1/2, was underexpressed by the chondrogenic medium in the sham-stimulated samples $(89 \%$ of that in ND controls) and overexpressed by CRET stimulation (33\% over differentiated controls CD).

\section{RF effects on SOX5 and SOX6 gene expression: RT-PCR assay}

Data in Figure 7 indicate that the chondrogenic medium can induce overexpression of genes SOX5 and SOX6, although only for SOX6 the differences over undifferentiated controls (ND) were statistically significant. On the other hand, the analysis of gene expression at the end of the 48 hours of RF exposure showed no significant differences with respect to the sham-exposed controls for either of the two genes studied. Inclusion in these experiments of RNA samples from human mature chondrocytes allowed validation of the above results by verifying that, as expected, these positive controls showed significantly high expression levels of genes SOX5 and SOX6 (2 and 3 orders of magnitude, respectively, over the $\mathrm{CD}$ differentiated samples; data no shown). a

\section{CD+CRET ND}

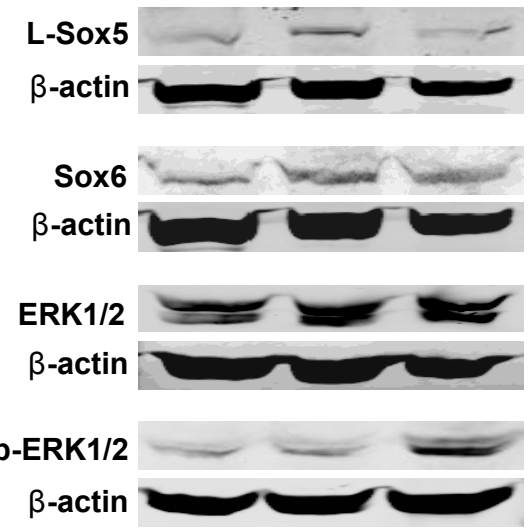

b

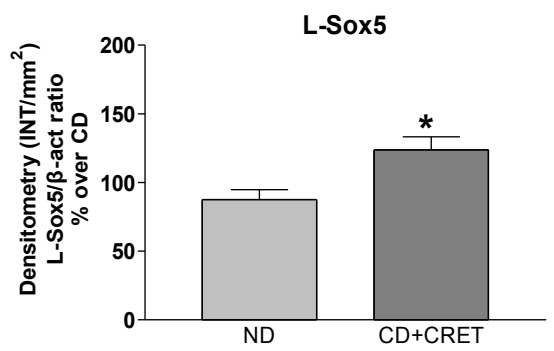

ERK1/2

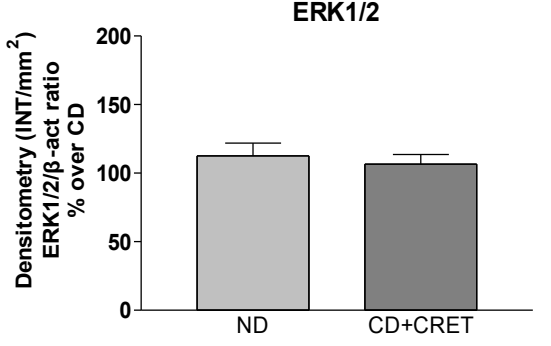

Sox 6

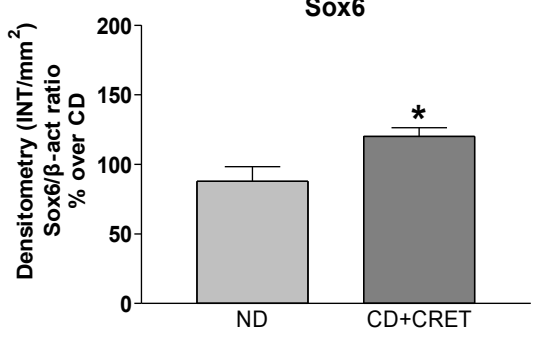

p-ERK $1 / 2$

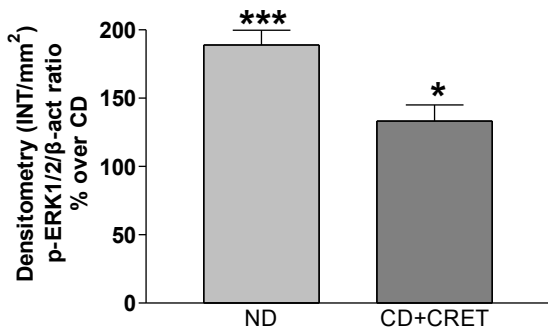

Figure 6: Western blot for L-Sox5, Sox6, ERK1/2 and p-ERK1/2 expression. $100 \mu$ g protein per lane. ND, CD and CD+CRET: same notation as in previous figures. (a) Representative blots. (b) Means \pm SD of protein expression (Protein/ $\beta$-actin ratio; INT/ $\mathrm{mm}^{2}$ : Intensity per $\mathrm{mm}^{2}$ ) in non-differentiated and in CRET-exposed, differentiated samples, normalized over sham-exposed, differentiated controls CD. Between 5 and 10 repeats per experimental group. ${ }^{*} 0.01 \leq p<0.05$; ${ }^{* * *} p<0.001$; Student's t test.

SOX5

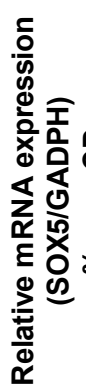

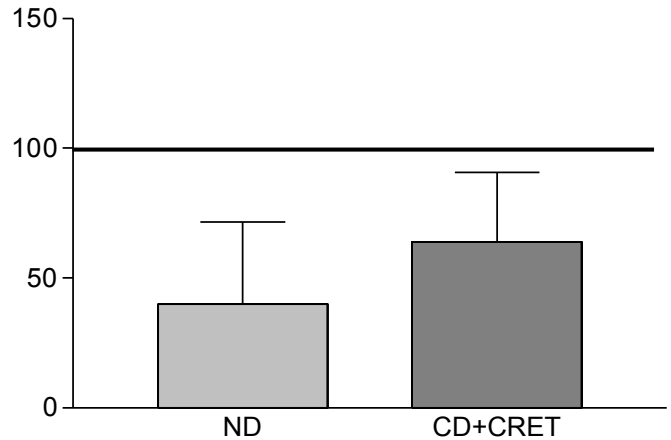

Figure 7: RT-PCR analysis of CRET effects on the expression of genes SOX5 and SOX 6 at the end of the 48 hours of RF- or sham-exposure. ND, CD and CD+CRET: same notations as in previous figures. Data are means \pm SD of 6 replicates, normalized over CD samples differentiated for 16 days and sham-exposed to CRET. ${ }^{*} 0.01 \leq p<0.05$. Student's $t$ test. 


\section{Discussion}

Previous studies by our group have shown that cyclic exposure to a subthermal dose of $448-\mathrm{kHz}$, sine wave electric current of the type applied in CRET therapy, can stimulate proliferation of ADSC cultures obtained from healthy human donors and grown in proliferating medium [6]. These data suggested that the beneficial effects on tissue repair and regeneration attributed to CRET therapies could be mediated in part by electrically-induced stimulation of the proliferation of stem cells present in damaged tissues.

The histological and physiological characteristics of the cartilage, with very low cell proliferation rate and a dense and fibrous extracellular matrix that hinders cell migration, restrict the repairing and regenerative capacities of this tissue in osteoarticular lesions or pathologies [23]. Several electrical therapies have been used as adjuvants in the treatment of joint cartilage injuries, and indications exist that electrical stimulation could potentiate cellular phenomena involved in chondrogenesis through promotion of stem cell differentiation [38]. Within this context, the present study investigates whether the electric component of the electrothermal stimulus applied in CRET therapies, besides of promoting proliferation of ADSC, could influence the early chondrogenic differentiation of these cells.

The XTT proliferation assay (Figure 2) revealed that chemical induction of chondrocytic differentiation also comports inhibition of cell proliferation (CD vs. ND). And, in contrast to the aforementioned proliferative effect of CRET in undifferentiated ADSC grown in proliferating medium [6], the same RF treatment exerts antiproliferative effects on ADSC undergoing chondrocytic differentiation (CD+CRET vs. CD). This could represent a basic indication that CRET may promote early chondrogenic differentiation.

The potential effects of the electrostimulation on chondrogenic differentiation were further assessed through quantification of the content of collagen and GAG, the major structural constituents of the cartilage extracellular matrix. As shown in Figures 3 and 4, the obtained results revealed that, compared with their sham-stimulated controls, the matrix of the samples intermittently exposed to the electric stimulus for 48 hours showed significantly increased levels of both, the cartilage-specific collagen type II, and GAG. These results add to the body of evidence that exposure to electric and/or magnetic signals at a variety of frequencies and durations, can induce significant changes in the synthesis and content of extracellular GAG and collagen during chondrogenic differentiation, both in vivo and in vitro [39]. In what regards specifically to the effects of electrostimulation at the intermediate frequency and radiofrequency ranges, our results are consistent in part with those reported by Wang et al. [40] showing that in vitro electrical stimulation at $60 \mathrm{kHz}$ and $20 \mathrm{mV} / \mathrm{cm}$ promotes extracellular matrix synthesis in bovine articular chondrocytes. These authors based their study on previous data showing that in vivo treatment with capacitive electric signals can promote nonunion fracture healing and spinal fusion [41].

During chondrogenic differentiation, the synthesis of extracellular matrix is regulated by the expression of transcription factors Sox 5 and Sox6 [42]. In humans, these proteins are encoded by the 20-membered SOX gene family (Sry-type HMG box). Sox 5 and Sox 6 regulate cartilage and cartilage-specific matrix formation, and maintain the chondrocyte phenotype in mature cartilage by activating the expression of several cartilage-specific genes, including those involved in collagen synthesis [43]. The extracellular matrix, in turn, regulates the signaling pathways to co-ordinate cartilage and bone formation [44]. Therefore, here we investigated the potential involvement of the RF effects on L-Sox5 and Sox6 protein expression in the above described electroinduced promotion of extracellular matrix synthesis. Both of the complementary techniques applied, immunofluorescence and immunoblot, revealed that, compared to sham-exposure, the electric treatment significantly increased L-Sox 5 and Sox6 expression (Figures 5 and 6).

As describe above, the results of the XTT assay (Figure 2) show that incubation in chondrogenic differentiating medium significantly inhibits cell proliferation (CD vs. ND) and that this antiproliferative effect is reinforced by RF electric stimulation (CD+CRET vs. CD). In relation to this, the immunoblot data in Figure 6 had shown that while the differentiating medium inhibits p-ERK1/2 expression, CRET induces overexpression of this activated form of MAPK, and that neither of both treatments affects the expression of total ERK1/2. These two data groups are coherent with each other, and are indicative of an inhibition of the ADSC cytoproliferative pathway by the differentiating medium, as well as of an electroinduced activation of ERK1/2, which is essential for progression of the chondrogenic differentiation. Indeed, the MAPK-ERK1/2 signaling pathway is a crucial regulator of cartilage-specific gene expression in a variety of chondroprogenitor and chondrogenic cell types [32]. It has also been reported that in bone marrow mesenchymal stem cells, chondrogenesis is accompanied with p-ERK1/2 overexpression and increased contents of collagen type II and glycosaminoglycans, and that underexpression of the ERK pathway members MEK1 and ERK1 induces underexpression of a variety of chondrogenic markers, including Sox 5 and Sox6 $[45,46]$. Within this context, the set of results reported here are indicative that the RF effects in promoting synthesis of chondrogenic extracellular matrix would be mediated, at least in part, by electro-induced overexpression of proteins L-Sox5 and Sox6, and activation of the MAPK-ERK1/2 pathway.

With regard to gene expression, the RT-PCR analysis revealed that incubation in chondrogenic medium increased the expression of genes SOX5 and SOX6 with respect to undifferentiated samples, though only for SOX6 the differences reached statistical significance. Although, as described above, the expression of transcription factors Sox 5 and Sox 6 was significantly increased at the end of 48 hours of CRET treatment, the expression of the corresponding genes, SOX5 and SOX6, did not show significant differences with respect to their controls, sham-exposed during the same time lapse. The possibility cannot be ruled out that an activation of genes SOX5 and SOX6 that had occurred during the early phases of the electric treatment, being therefore undetectable at the end of the exposure interval, was responsible for the increase in the expression of the corresponding transcription factors, observed after 48 hours of CRET treatment. However, since the understanding of the way in which the expression of those transcription factors is regulated during chondrogenesis remains incomplete [47,48], the potential effects of CRET on gene expression cannot be sufficiently elucidated on the basis of the present results and need to be further investigated. On the other hand, CRET stimulation might also influence epigenetic mechanisms that regulate gene expression during chondrogenic differentiation. Such mechanisms include, among others, methylation of cartilage-specific promoter genes, or acetylation, methylation, phosphorylation and SUMOylation of histones, which depending on the case, are able of promoting or repressing the transcriptional activation of chondrogenic genes such as SOX9 (see [49] for a review). In fact, in contrast to the observations in this paper, ERK1/2 signaling pathway has shown able to facilitate chondrogenic differentiation in the dental pulp stem cells (DPSCs) [50], what indicates that MAPK pathway activation might play distinct roles in the differentiation of stem cells from different lineages. As epigenetic mechanisms play a pivotal role in 
the stem cell fate commitment, it is possible that epigenetic factors are involved in the herein reported effects of CRET on the chondrogenic differentiation of ADSC. For instance, histone methyltransferase G9a is able to inactivate MAPK signaling via negative regulation of the dual specificity phosphatase-4 (DUSP4) in head and neck squamous cell carcinoma [51]. In addition, pharmacological inhibition of G9a has also been found to inactivate MAPK signaling in non-small-cell lung cancer (NSCLC) [52]. At the molecular level, the main function of G9a and its partner protein GLP is depositing $\mathrm{H} 3 \mathrm{~K} 9 \mathrm{me} 2 / 3$ at the euchromatin loci [53]. Apart from being a histone modifier, G9a recently has also been found to play a role in the maintenance of imprinted DNA methylation in embryonic stem cells [54]. Therefore, the study of the electrical stimulation effects on the patterns of $\mathrm{G} 9 \mathrm{a}$ associated $\mathrm{H} 3 \mathrm{~K} 9 \mathrm{me} 2 / 3$ as well as on DNA methylation, might elucidate the epigenetic mechanisms potentially involved in the lineage commitment of CRET-exposed ADSC during the differentiation process.

In sum, together with previously reported results [6], the present dataset provides support to the hypothesis that the electric component of the electrothermal stimulus applied in CRET therapy could promote tissue repair and regeneration. Concerning cartilage tissue, at initial repair stages the electric stimulation could promote stem cell proliferation in the injured tissues [6], whereas later, at early or intermediate differentiational stages, the electric treatment may stimulate chondrogenesis through overexpression of transcription factors such as Sox 5 and Sox6, and activation of p-ERK1/2. These effects would result in the observed increases in the synthesis and content of type II collagen and GAG in the extracellular matrix of the CRET-exposed cultures. This opens the possibility that radiofrequency currents can be successfully applied in early/intermediate phases of chondrogenic repair and regeneration. Nevertheless, important aspects of the in vitro response to electrical stimulation are still to be investigated, especially at advanced stages of the chondrogenic differentiation. Furthermore, the effects on the cartilaginous tissue of the joint action of the electric stimulation in combination with the concomitant hyperthermia, as applied to the patient in electrothermal CRET therapies, is yet to be elucidated.

\section{Author's Contribution}

Conceived and designed the experiments: MLHB, AU. Performed the experiments:MLHB. Analyzed the data: MLHB, CA, MAMG, MAT, AU. Designed and developed the algorithms for image-based quantification of collagen: $C A$. Performed the RT-PCR analysis: MAMG. Wrote the paper: MLHB, MAT, AU.

\section{Funding}

This work was financially supported by Fundación para la Investigación Biomédica del Hospital Ramón y Cajal (Project FiBio-HRC No. 2012/0032). The founder had no role in the study design, data collection and analysis, decision to publish or preparation of the manuscript.

\section{Acknowledgments}

The authors thank Ms. Silvia Sacristán and Ms. Elena Toledano-Macías (both Hospital Universitario Ramón y Cajal-IRYCIS) for helpful technical advice and assistance.

\section{Declaration}

The authors declare no conflict of interest.

\section{References}

1. Li X, Kolega J (2002) Effects of direct current electric fields on cell migration and actin filament distribution in bovine vascular endothelial cells. J Vasc Res 39: 391-404. [PubMed]

2. Cho MR, Thatte HS, Lee RC, Golan DE (1994) Induced redistribution of cell surface receptors by alternating current electric fields. FASEB J 8: 771-776. [PubMed]

3. Ross CL (2017) The use of electric, magnetic, and electromagnetic field for directed cell migration and adhesion in regenerative medicine. Biotechnol Prog 33: 5-16. [PubMed]

4. Titushkin IA, Rao VS, Cho MR (2004) Mode- and cell-type dependent calcium responses induced by electrical stimulus. IEEE Trans Plasma Sci 32: 1614-1619.

5. Clark CC, Wang W, Brighton CT (2014) Up-regulation of expression of selected genes in human bone cells with specific capacitively coupled electric fields. J Orthop Res 32: 894-903. [PubMed]

6. Hernández-Bule ML, Paíno CL, Trillo MA, Úbeda A (2014) Electric stimulation at $448 \mathrm{kHz}$ promotes proliferation of human mesenchymal stem cells. Cell Physiol Biochem 34: 1741-1755. [PubMed]

7. Hernández-Bule ML, Martínez-Botas J, Trillo MA, Paíno CL, Úbeda A (2016) Antiadipogenic effects of subthermal electric stimulation at $448 \mathrm{kHz}$ on differentiating human mesenchymal stem cells. Mol Med Rep 13: 3895-3903. [PubMed]

8. Ciombor DM, Aaron RK (2005) The role of electrical stimulation in bone repair. Foot Ankle Clin 10: 579-593. [PubMed]

9. Ashrafi M, Alonso-Rasgado T, Baguneid M, Bayat A (2017) The efficacy of electrical stimulation in lower extremity cutaneous wound healing: a systematic review. Exp Dermatol 26: 171-178. [PubMed]

10. Willand MP, Nguyen MA, Borschel GH, Gordon T (2016) Electrical stimulation to promote peripheral nerve regeneration. Neurorehabil Neural Repair 30: 490-496.

11. Tuszynski JA, Wenger C, Friesen DE, Preto J (2016) An overview of subcellular mechanisms involved in the action of TTFields. Int $\mathrm{J}$ Environ Res Public Health 12: E1128. [PubMed]

12. Garland D, Holt P, Harrington JT, Caldwell J, Zizic T, et al. (2007) A 3-month, randomized, double-blind, placebo-controlled study to evaluate the safety and efficacy of a highly optimized, capacitively coupled, pulsed electrica stimulator in patients with osteoarthritis of the knee. Osteoarthritis Cartilage 15: 630-637. [PubMed]

13. Fary RE, Carroll GJ, Briffa TG, Gupta R, Briffa NK (2008) The effectiveness of pulsed electrical stimulation (E-PES) in the management of osteoarthritis of the knee: a protocol for a randomised controlled trial. BMC Musculoskele Disord 9: 18. [PubMed]

14. Maurer P, Block JE, Squillante D (2008) Intradiscal electrothermal therapy (IDET) provides effective symptom relief in patients with discogenic low back pain. J Spinal Disord Tech 21: 55-62. [PubMed]

15. Rohof O (2012) Intradiscal pulsed radiofrequency application following provocative discography for the management of degenerative disc disease and concordant pain: a pilot study. Pain Pract 12: 342-349.

16. Parolo E, Honesta MP (1998) HCR 900: hyperthermia by capacitive and resistive energy transfer in the treatment of acute and chronic muscularskeletal injuries. La Riabilitazione $31: 81-83$.

17. Takahashi K, Suyama T, Onodera M, Hirabayashi S, Tsuzuki N, et al. (1999) Clinical effects of capacitive electric transfer hyperthermia for lumbago. J Phys Ther Sci 11: 45-51.

18. Mondardini P, Tanzi R, Verardi L, Briglia S, Maione A, et al. (1999) New methods for the treatment of traumatic muscle pathology in athletes: C.R.E.T therapy. Excerpt from medicina dello sport 52: 201-213.

19. Ganzit GP (2000) New methods in the treatment of joint-muscular pathologies in athletes: CRET therapy. Excerpt from Medicina dello sport 53: 361-367.

20. Melegati G, Volpi P, Tornese D, Mele G (1999) Rehabilitation in tendinopathies Sports Traumatol Rel Res 21: 66-83.

21. Ganzit CP, Gabriele G (2001) CRET therapy in treatment of tendinopathies II Medico Sportivo Suppl No.1.

22. Melegati G, Tornese D, Bindi M (2000) The use of CRET therapy ankle sprains. La Riabilitazione 33: 163-167.

23. Mauck RL, Martínez-Díaz GJ, Yuan X, Tuan RS (2007) Regional multilineage differentiation potential of meniscal fibrochondrocytes: implications for meniscus repair. Anat Rec (Hoboken) 290: 48-58. [PubMed] 
Citation: Hernández-Bule ML, Trillo MÁ, Martínez-García MÁ, Abilahoud C, Úbeda A (2017) Chondrogenic Differentiation of Adipose-Derived Stem Cells by Radiofrequency Electric Stimulation. J Stem Cell Res Ther 7: 407. doi: 10.4172/2157-7633.1000407

24. Tan L, Ren Y, van Kooten TG, Grijpma DW, Kuijer R (2015) Low-intensity pulsed ultrasound (LIPUS) and pulsed electromagnetic field (PEMF) treatments affect degeneration of cultured articular cartilage explants. Int Orthop 39: 549-557. [PubMed]

25. Aaron RK, Ciombor DMK (1996) Acceleration of experimental endochondral ossification by biophysical stimulation of the progenitor cell pool. J Orth Res 14: 582-589. [PubMed]

26. Creecy CM, O'Neill CF, Arulanandam BP, Sylvia VL, Navara CS, et al. (2013) Mesenchymal stem cell osteodifferentiation in response to alternating electric current. Tissue Eng Part A 19: 467-474. [PubMed]

27. Taghian T, Narmoneva DA, Kogan AB (2015) Modulation of cell function by electric field: a high-resolution analysis. J R Soc Interface 6: 20150153. [PubMed]

28. Aaron RK, Boyan BD, Ciombor DM, Schwartz Z, Simon BJ (2004) Stimulation of growth factor synthesis by electric and electromagnetic fields. Clin Orthop Relat Res 419: 30-37. [PubMed]

29. Meng X, Arocena M, Penninger J, Gage FH, Zhao M, et al. (2011) PI3K mediated electrotaxis of embryonic and adult neural progenitor cells in the presence of growth factors. Exp Neurol 227: 210-217. [PubMed]

30. Hronik-Tupaj M, Rice WL, Cronin-Golomb M, Kaplan DL, Georgakoudi I (2011) Osteoblastic differentiation and stress response of human mesenchymal stem cells exposed to alternating current electric fields. Biomed Eng Online 26: 9. [PubMed]

31. Teven CM, Greives M, Natale RB, Su Y, Luo Q, et al. (2012) Differentiation of osteoprogenitor cells is induced by high-frequency pulsed electromagnetic fields. J Craniofac Surg 23: 586-593. [PubMed]

32. Bobick BE, Kulyk WM (2008) Regulation of cartilage formation and maturation by mitogen-activated protein kinase signaling. Birth Defects Res $C$ Embryo Today 84: 131-154. [PubMed]

33. Ikeda T, Kawaguchi H, Kamekura S, Ogata N, Mori Y, et al. (2005) Distinct roles of Sox5, Sox6, and Sox9 in different stages of chondrogenic differentiation. J Bone Miner Metab 23: 337-340. [PubMed]

34. Hamid AA, Idrus RB, Saim AB, Sathappan S, Chua KH (2012) Characterization of human adipose-derived stem cells and expression of chondrogenic genes during induction of cartilage differentiation. Clinics (Sao Paulo) 67: 99-106. [PubMed]

35. Ruzon M, Rubner Y (1997) https://www2.eecs.berkeley.edu/Research/ Projects/CS/vision/bsds/code/Util/Lab2RGB.m Accessed 11 July 2017.

36. Hernández-Bule ML, Trillo MA, Cid MA, Leal J, Ubeda A (2007) In vitro exposure to $0.57-\mathrm{MHz}$ electric currents exerts cytostatic effects in HepG2 human hepatocarcinoma cells. Int J Oncol 30: 583-592. [PubMed]

37. Yamashita S, Miyaki S, Kato Y, Yokoyama S, Sato T, et al. (2012) L-Sox5 and Sox6 proteins enhance chondrogenic miR-140 microRNA expression by strengthening dimeric Sox9 activity. J Biol Chem 287: 22206-22215. [PubMed]

38. Chen CH, Lin YS, Fu YC, Wang CK, Wu SC, et al. (1985) Electromagnetic fields enhance chondrogenesis of human adipose-derived stem cells in a chondrogenic microenvironment in vitro. J Appl Physiol 114: 647-655. [PubMed]

39. Ciombor DM, Lester G, Aaron RK, Neame P, Caterson B (2002) Low frequency EMF regulates chondrocyte differentiation and expression of matrix proteins. J Orthop Res 20: 40-50. [PubMed]
40. Wang W, Wang Z, Zhang G, Clark CC, Brighton CT (2004) Up-regulation of chondrocyte matrix genes and products by electric fields. Clin Orthop Relat Res 427 Suppl: S163-173. [PubMed]

41. Goodwin CB, Brighton CT, Guyer RD, Johnson JR, Light KI, et al. (1999) A double-blind study of capacitively coupled electrical stimulation as an adjunct to lumbar spinal fusions. Spine 24: 1349-1357. [PubMed]

42. Lefebvre V, Li P, de Crombrugghe B (1998) A new long form of Sox5 (L-Sox5) Sox6 and Sox9 are coexpressed in chondrogenesis and cooperatively activate the type II collagen gene. EMBO J 17:.5718-33. [PubMed]

43. Liu CF, Lefebvre $V(2015)$ The transcription factors SOX9 and SOX5/SOX6 cooperate genome-wide through super-enhancers to drive chondrogenesis. Nucleic Acids Res 43: 8183-8203. [PubMed]

44. Gao Y, Liu S, Huang J, Guo W, Chen J, et al. (2014) The ECM-cell interaction of cartilage extracellular matrix on chondrocytes. Biomed Res Int 2014: 648459. [PubMed]

45. Smits P, Li P, Mandel J, Zhang Z, Deng JM, et al. (2001) The transcription factors L-Sox 5 and Sox6 are essential for cartilage formation. Dev Cell 1: 277-290. [PubMed]

46. Bobick BE, Matsche Al, Chen FH, Tuan RS (2010) The ERK5 and ERK1/2 signaling pathways play opposing regulatory roles during chondrogenesis of adult human bone marrow-derived multipotent progenitor cells. J Cell Physiol 224: 178-186. [PubMed]

47. Akiyama H, Chaboissier MC, Martin JF, Schedl A, de Crombrugghe B (2002) The transcription factor Sox9 has essential roles in successive steps of the chondrocyte differentiation pathway and is required for expression of Sox 5 and Sox6. Genes Dev 16: 2813-2828. [PubMed]

48. Hagiwara N (2011) Sox6, Jack of all trades: A versatile regulatory protein in vertebrate development. Dev Dyn 240: 1311-1321. [PubMed]

49. Hata K (2015) Epigenetic regulation of chondrocyte differentiation. Japanese Dental Science Review 51: 105-113.

50. Ba P, Duan X, Fu G, Lv S, Yang P, Sun Q (2017) Differential effects of $p 38$ and Erk $1 / 2$ on the chondrogenic and osteogenic differentiation of dental pulp stem cells. Mol Med Rep 16: 63-68. [PubMed]

51. Li KC, Hua KT, Lin YS, Su CY, Ko JY, et al. (2014) Inhibition of G9a induces DUSP4-dependent autophagic cell death in head and neck squamous cell carcinoma. Mol Cancer 13: 172. [PubMed]

52. Huang T, Zhang P, Li W, Zhao T, Zhang Z, et al. (2017) G9A promotes tumor cell growth and invasion by silencing CASP1 in non-small-cell lung cancer cells. Cell Death Dis 6: e2726. [PubMed]

53. Tachibana M, Sugimoto K, Fukushima T, Shinkai $Y$ (2001) Set domaincontaining protein, G9a, is a novel lysine-preferring mammalian histone methyltransferase with hyperactivity and specific selectivity to lysines 9 and 27 of histone H3. J Biol Chem 276: 25309-17. [PubMed]

54. Zhang T, Termanis A, Özkan B, Bao XX, Culley J, et al. (2016) G9a/GLP complex maintains imprinted DNA methylation in embryonic stem cells. Cell Rep 15: 77-85. [PubMed] 